\title{
Four-dimensional dynamic flow measurement by holographic particle image velocimetry
}

\author{
Ye Pu and Hui Meng
}

\begin{abstract}
The ultimate goal of holographic particle image velocimetry (HPIV) is to provide space- and time-resolved measurement of complex flows. Recent new understanding of holographic imaging of small particles, pertaining to intrinsic aberration and noise in particular, has enabled us to elucidate fundamental issues in HPIV and implement a new HPIV system. This system is based on our previously reported off-axis HPIV setup, but the design is optimized by incorporating our new insights of holographic particle imaging characteristics. Furthermore, the new system benefits from advanced data processing algorithms and distributed parallel computing technology. Because of its robustness and efficiency, for the first time to our knowledge, the goal of both temporally and spatially resolved flow measurements becomes tangible. We demonstrate its temporal measurement capability by a series of phase-locked dynamic measurements of instantaneous three-dimensional, three-component velocity fields in a highly three-dimensional vortical flow-the flow past a tab. () 2005 Optical Society of America
\end{abstract}

OCIS codes: $290.5850,090.0090,120.7250,110.6880$.

\section{Introduction}

Turbulent flow is a highly complex and yet ubiquitous physical phenomenon that we know little about. The understanding, modeling, and control of turbulence requires proper experimental tools to reveal the fluctuating three-dimensional (3D) vorticity at a wide range of time and length scales, which has posed an overwhelming challenge to modern technology in many aspects. The recently matured particle image velocimetry (PIV) technique only partially fulfills this need by providing two-component ${ }^{1,2}$ or three-component ${ }^{3,4}$ velocity measurement on a two-dimensional (2D) plane. Further explorations ${ }^{5,6}$ attempted to expand PIV capabilities to cover a volumetric domain by rapidly scanning the volume plane by plane. However, instantaneous, full-field 3D measurement with sufficient spatial resolution and dynamic range is beyond the reach of conventional PIV.

When this research was performed the authors were with the Laser Flow Diagnostics Laboratory, Department of Mechanical and Aerospace Engineering, State University of New York at Buffalo, Buffalo, New York 14260. Y. Pu (yepu@sunoptics.caltech.edu) is now with the Department of Electrical Engineering, California Institute of Technology, Pasadena, California 91125. The e-mail address for H. Meng is huimeng@eng.buffalo.edu.

Received 21 April 2005; revised 7 September 2005; accepted 8 September 2005.

0003-6935/05/367697-12\$15.00/0

(C) 2005 Optical Society of America
Holographic particle image velocimetry (HPIV) is a full-field, instantaneous, 3D flow diagnostics tool that holds the promise to provide unprecedented experimental capability for fluid dynamics research. Using holography, HPIV records and reconstructs two 3D images, at distinct moments in time, for the small particles dispersed in the fluid flow under investigation. The recording is considered instantaneous because the process completes as fast as a laser pulse (usually in a $10 \mathrm{~ns}$ range). From the displacements of particles $(\Delta \mathbf{x})$ during the time interval $(\Delta t)$ between the two recording moments, the fluid velocities are deduced as $\mathbf{u}=\Delta \mathbf{x} / \Delta t$. Extension of the measurement in the temporal domain (by recording multiple holograms) leads to cinematic HPIV, which provides both space- and time-resolved velocity field measurements.

HPIV can be implemented in either in-line or offaxis configurations. Because of its simplicity, in-line holography attracted earlier application of particle holography. ${ }^{7-11}$ However, because of its excessive intrinsic speckle noise in the reconstructed image, ${ }^{12}$ inline holography was restricted to measurements of sparsely dispersed particles.10,11 Thus later developments in HPIV have concentrated on off-axis holography, which shows significantly improved imaging signal-to-noise ratio (SNR) over the in-line versions, allowing for much higher seeding densities and thus greater spatial resolutions. ${ }^{13,14}$ Indeed, most of the recent film-based HPIV studies use off-axis holography. ${ }^{13-18}$ On the other hand, the newly emerged digital 
HPIV is mostly implemented in an in-line version due to poor resolutions of current electronic imagers. Recent reviews in the area of HPIV can be found in Refs. 19 and 20.

Despite the large variety of designs in practice, fundamental knowledge about the physics in HPIV is nevertheless rather limited. In particular, our understanding about the imaging characteristics of particle holography and the associated speckle noise is still at a rudimentary level. The lack of such knowledge hinders the objective justification of a particular design and the reliable estimation of its accuracies and capabilities. The rationales for common HPIV designs are mostly based on engineering convenience rather than quantitative analysis. Such systems inevitably suffer from great operational difficulties and unrepeatable results. Consequently, four-dimensional HPIV measurement, a natural and highly anticipated extension to the HPIV technique, remains an elusive goal.

In view of this lack of knowledge, we have developed theories of the signal and noise properties for particle holography. ${ }^{21,22}$ We show that imperfect 3D particle images formed by the wavefront reconstruction of the light scattering and the associated speckle noises hold the primary responsibility for the limit in the accuracy and capacity of HPIV. These two issues are intrinsic to the physical process in HPIV. Their negative effects can be alleviated only by better choices of system parameters but never be eliminated.

In this paper we first provide a synopsis of the imaging and noise properties of particle holography, based on the determining factors in the capability of HPIV. These lead to guidelines for direct evaluation of HPIV designs. We then report our latest development in off-axis HPIV technology. We demonstrate the temporal potential of HPIV with a phase-locked measurement of a flow passing a wall-mounted tab, which reveals a sequence of a vortex shedding process in a complete cycle. This experiment shows that HPIV holds great promise for four-dimensional (3D space plus time) measurements of fluid flow.

\section{Basic Configurations and Fundamental Issues in Holographic Particle Image Velocimetry}

Light scattering by small particles plays a paramount role in HPIV, since it is the signal that the hologram records and reconstructs. As will be shown in later discussions, the scattering wavefront from each particle and the spatial dispersion of the scattering sources (particles) lead to two fundamental issues in HPIV: intrinsic aberration and intrinsic noise. Because of this importance of scattering phenomena, we categorize HPIV configurations by the recording angle $\left(\theta_{H}\right)$, i.e., the angle between the normal vector of the recording plane and the propagation vector of the object-illuminating light. Figure 1 illustrates these HPIV configurations, while also stressing the critical role of polarization. There are three major categories of HPIV configurations: forward (or near-forward) recording $\left(\theta_{H} \approx 0^{\circ}\right.$ ), backward (or near-backward) recording $\left(\theta_{H} \approx 180^{\circ}\right)$, and $90 \mathrm{deg}$ recording $\left(\theta_{H}\right.$

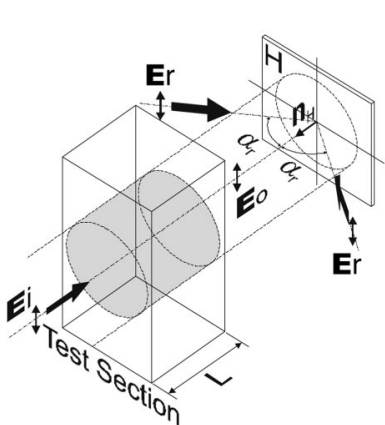

(a)

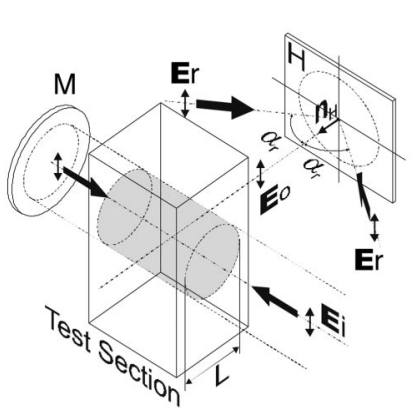

(c)

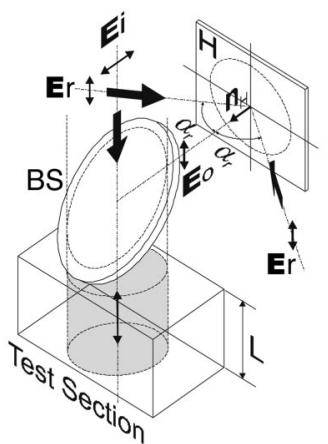

(b)

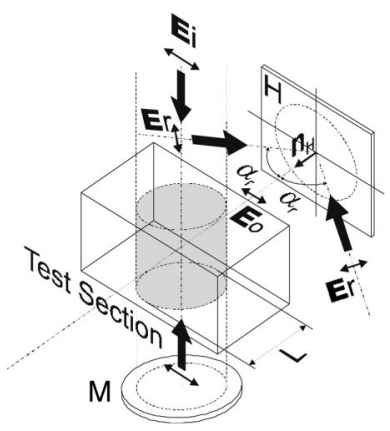

(d)
Fig. 1. Optical configurations for off-axis HPIV. (a) Forward scattering; (b) backward scattering; (c) 90 deg scattering, vertical polarization configuration; (d) $90 \mathrm{deg}$, horizontal polarization configuration. $\mathbf{E}_{i}$, illuminating wave; $\mathbf{E}_{r}$, reference wave; H, hologram plane; $\mathbf{n}_{H}$, normal vector of holographic plane; $\theta_{H}$, recording angle; $\alpha_{r}$, reference angle; M, mirror; BS, beam splitter.

$\approx 90^{\circ}$ ). On the basis of the polarizations of the reference and object waves, 90 deg recording is further classified as a vertical polarization configuration, where the polarizations of the object wave and the reference waves are parallel everywhere, and a horizontal polarization configuration, where the polarizations of the object wave and the reference waves are not parallel to each other but share a parallel component.

In the $90 \mathrm{deg}$ recording configurations [Figs. 1(c) and $1(\mathrm{~d})$ ], an optional backreflection mirror can be employed to boost the scattering intensity. The hologram records the sum of two wavefronts scattered from both illuminations, whereby the intensity of the object wave is approximately doubled. The reconstructed image is a coherent sum of two overlapping images containing an intrinsic aberration from each. This mirror, however, should be excluded when very small tracer particles (comparable or smaller than the recording wavelength) are used due to the spatial intensity fluctuation of the standing wave created by the mirror.

Optical access at nonorthogonal angles is certainly possible and was successfully employed for the measurement in gas flow. ${ }^{13}$ However, without special optical treatment, liquid media and the walls of the flow facility can cause severe aberrations and distortions, 
which seriously damage the signal integrity. For this reason, we favor the chosen three orthogonal angles.

In Subsections 2.A and 2.B we discuss the intrinsic aberration and intrinsic speckle noise in off-axis particle holography and the effects of particular HPIV configurations on these issues.

\section{A. Intrinsic Aberration}

In an ideal particle holography system, for each particle the hologram records spherical waves converging at the center of the particle without aberration. This paradigm is often the assumption of many HPIV developments. We have shown in recent work, ${ }^{21}$ however, that even a perfect hologram (aperture limited) is unable to faithfully reconstruct the 3D image of a particle (i.e., a sphere). The reconstructed 3D image presents dramatic changes in image morphologies depending on the recording angle $\theta_{H}$ and particle size $d_{p}$. This imperfection is a result of highly uneven intensity and phase distribution of the scattering wavefront ${ }^{23}$ across the hologram aperture, and we treat such imperfection as a special type of aberration.

The consequence of the intrinsic aberration is highly complex 3D particle image intensity distributions, as well as bodily deviations of the particle images from their true locations, causing great difficulties to identify and locate the particles precisely. The intrinsic aberration also degrades the SNR of the reconstruction images and thus reduces the information capacity in HPIV (to be discussed in Section 3). Such degradation can be quantified with the image integrity $\gamma(0<\gamma \leq 1)$, defined by the ratio of the actual image intensity to the image intensity of an undistorted spherical wave with the same energy. A perfect particle image has $\gamma=1$, while aberrations result in lower image integrity values.

Numerical simulations ${ }^{21}$ show that the intrinsic aberration varies significantly with $\theta_{H}$, and the negative effect of intrinsic aberration on the measurement accuracy is minimal in a $90 \mathrm{deg}$ configuration. The relationship between the intrinsic aberration and the particle size clearly calls for use of smaller particles since the scattered wave more closely resembles a spherical wave. However, because of the constraints of optical configuration and available laser power, the size of the particles used in most reported HPIV systems often ranges from 10 to $50 \mu \mathrm{m}$, with exceptions in Refs. $13(\sim 0.5 \mu \mathrm{m})$ and 18 $(\sim 1-3 \mu \mathrm{m})$. Significant intrinsic aberration is expected at this size range.

Although most practical cases are dominated by extrinsic aberrations from imperfect optics and holograms, these aberrations can be eliminated through phase-conjugate reconstruction ${ }^{13}$ or complex correlation. ${ }^{24}$ Conversely, the intrinsic aberration cannot be removed. Therefore the measured particle position is always subject to a deviation due to the intrinsic aberration, although in special cases the particle displacement may not be subject to deviation. ${ }^{24}$ The role of the intrinsic aberration in the measurement of particle displacements becomes more subtle in innovations where phase aberrations are canceled through complex correlation of the local scattered field. ${ }^{24}$ Although the accuracy of displacement measurements has been greatly improved through the removal of the intrinsic phase aberration, the remaining amplitude modulation could still be severe enough to prevent the reach of submicrometer accuracy.

\section{B. Intrinsic Speckle Noise}

Speckle noise is intrinsic in coherent imaging. Because of the self-interference of a large amount of reconstructed waves from particle images dispersed in the $3 \mathrm{D}$ space, the speckle noise problem in particle holography is especially severe and has long been the major obstacle for HPIV to reach a high spatial sampling rate. The SNR in a reconstructed holographic image can be expressed as ${ }^{25}$

$$
\frac{I_{0}}{\sigma_{N}}=\frac{I_{0} /\left\langle I_{N}\right\rangle}{\sqrt{1+2 I_{0} /\left\langle I_{N}\right\rangle}},
$$

where $I_{0}$ is the focal intensity of the aberration-free (diffraction-limited) particle image, and we have shown in Ref. 22 that in particle holography,

$$
\frac{I_{0}}{\left\langle I_{N}\right\rangle}=\frac{\pi \tan ^{2} \Omega}{\lambda^{2} n_{s} L} .
$$

Given a proper holographic recording and the minimum SNR required by a particular algorithm for correct extraction of particle positions, the intrinsic speckle noise is the essential limiting factor for the maximum achievable number density of particles (seeding density). Equation (2) suggests that the seeding density $n_{s}$ and the axial depth $L$ must satisfy the following relationship:

$$
n_{s} L \leq \frac{\pi \tan ^{2} \Omega}{\lambda^{2}\left[\frac{I_{0}}{\left\langle I_{N}\right\rangle}\right]_{\min }},
$$

assuming spherical-wave reconstructions and infinitesimal sampling pixels (i.e., the best case). Analyses in Ref. 22 also show that, statistically, the speckle noise has very similar spectral characteristics to particle images regardless of the optical configuration. Consequently, 2D or 3D filtering is ineffective in the removal of speckle noise. Therefore inequality (3) represents a fundamental limit in the information a hologram can deliver. In later discussion, we refer to this limit as the information capacity of a hologram.

In practice, where particle images contain intrinsic aberrations and the sampling pixel has a finite size, this limit is reduced by a factor of $\gamma / \mathcal{M}$, where $M$ is the ratio of the pixel size to the mean speckle size. ${ }^{26}$

Inequality (3) stresses the importance of the angular aperture $\Omega$. In a forward-scattering configuration, $\Omega$ is usually determined by the size of the seeding particle. In configurations utilizing other scattering 
angles, $\Omega$ is limited by the recording and reconstruction optics, which can be much larger. Hence, even without the superposition of the reference wave, inline configurations often possess an inferior SNR than other designs due to its typically smaller angular aperture (which, however, can be improved by using smaller particles). Inequality (3) also reveals the importance of our controlling the depth dimension $L$ : Given a HPIV configuration and the SNR requirement, higher spatial resolution can be achieved at the expense of reduced $L$. This suggests that $90 \mathrm{deg}$ recording is again in favor because $L$ can be easily controlled by adjusting the illuminating beam to illuminate only the region of interest.

\section{Capacity and Accuracy of Holographic Particle Image Velocimetry}

The capability of HPIV system in flow measurements is bound by two factors: seeding density, which determines the spatial data sampling rate, and particle position uncertainty, which sets the primary limit in the accuracy and dynamic range of the measurement.

The number density of tracer particles determines the spatial resolution of the flow velocity field and hence the size of the smallest resolvable flow structures. The depth of the test volume $L$ determines the largest eddy size that the measurement can capture. For a uniform particle dispersion, we take the mean free distance between particles as the smallest eddy size: $\eta=\left(3 / 4 \pi n_{s}\right)^{1 / 3}$. Let us assume the integral length to be $L$ and the Kolmogorov length scale (the smallest intrinsic scale of turbulent motions) to be $\eta$; the maximum flow Reynolds number resolvable in HPIV measurement is ${ }^{27}$

$$
\operatorname{Re}_{\max }=\left(\frac{L}{\eta}\right)^{4 / 3}=\left(\frac{4 \pi n_{s} L^{3}}{3}\right)^{4 / 9} .
$$

Equation (4) shows that, given the information capacity $\left(n_{s} L\right)$ of a HPIV system, $\operatorname{Re}_{\max }$ scales almost linearly with $L$. As an example, let us consider an optimal case where $\gamma / M=1$ and $\tan \Omega=1$ is achieved, and we require a minimum SNR of $5\left(\left[I_{0} /\left\langle I_{N}\right\rangle\right]_{\min }=50\right)$. The information capacity is therefore $2.2 \times 10^{5} \mathrm{~mm}^{-2}$ (at $\lambda=532 \mathrm{~nm}$ ). At $L$ $=50 \mathrm{~mm}$, we are able to achieve $n_{s}=4.4$ $\times 10^{3} \mathrm{~mm}^{-3}$ and resolve $\operatorname{Re}_{\max }=1.45 \times 10^{4}$. At $L$ $=10 \mathrm{~mm}$, on the other hand, we are able to use a higher seeding density of $n_{s}=2.2 \times 10^{4} \mathrm{~mm}^{-3}$ but can only resolve $\operatorname{Re}_{\max }=3.48 \times 10^{3}$.

The uncertainty in the reconstructed particle position $\left(\varepsilon_{p}\right)$ is specific to the optical setup, particle size, particle relative refractive index, and the specific algorithm to extract the particle position. The flow velocity extracted from displacement $(\Delta x)$ between particles in the double exposures inherits its uncertainty $\left(\varepsilon_{v}\right)$ from the uncertainty in the particle positions $\left(\varepsilon_{p}\right)$. Uncertainties in $\Delta t$ are usually negligible owing to the picosecond accuracy in typical modern timing devices. Thus, for velocities obtained from individually paired particles,

$$
\varepsilon_{v}=\sqrt{\varepsilon_{p}^{2}+\varepsilon_{p}^{2}} / \Delta t=\sqrt{2} \varepsilon_{p} / \Delta t .
$$

In most practical cases, one can assume small displacement gradients within the local domain where the correlation is performed. Thus, at a cost of lowered spatial resolution, the displacement uncertainty can be roughly reduced to $1 / \sqrt{m}$ by calculating the mean velocity from a group of $m$ particles. ${ }^{28}$

For monodispersed particles, the system errors due to Mie scattering and the holography process do not significantly effect the accuracy of particle displacements, since most of the deviations in particle positions cancel out. If the particle size is nonuniform, the uncertainty in particle size is translated into the uncertainty of the particle positions and displacements.

In optical reconstruction, other sources of errors include mechanical misalignments ${ }^{29}$ and vibrations during the scanning of the reconstructed image field. Furthermore, the shrinkage of the holographic emulsion due to chemical processing also drastically degrades the reconstructed images and introduces significant measurement error. The above analysis, nonetheless, lays out the theoretical limit in the capacity and accuracy of HPIV. In particular, these limits also apply in digitally recorded holograms where emulsion shrinkage, mechanical misalignments, and vibrations are eliminated.

\section{Gemini Holographic Particle Image Velocimetry System}

One of the established HPIV systems is the Gemini system $^{14}$ developed in our laboratory. It employs 90 deg scattering and off-axis holography. The engineering intuition behind this design turned out to be effective; the Gemini system has proven to be a robust HPIV instrument and conforms to the guidelines laid out by the theory. The system consists of two major components: (1) a holographic recording and reconstruction subsystem and (2) a data processing subsystem. Over the past few years, this system has evolved significantly, incorporating the latest understanding of particle holography and advanced parallel computing technology into its implementations. In the following, we present this integrated system in its optical configuration, data processing algorithms, and distributed parallel computing cluster.

\section{A. Optical Configuration}

We have previously reported the optical configuration of the holographic recording and reconstruction subsystem in the Gemini HPIV in Ref. 14. Here we briefly describe the optical configuration of the Gemini system. Figure 2(a) illustrates the optical configuration for the HPIV recording stage. We use an injection-seeded, dual-cavity Nd:YAG laser (Spectra-Physics PIV-400) in this system, which gives a pair of temporally and spatially separated laser pulses, each of $8 \mathrm{~ns}$ duration. The pulse separation time $\Delta t$ is adjusted according to the estimated flow speed. The two laser pulses are split into two parts by the beam splitters. Eighty percent of the 


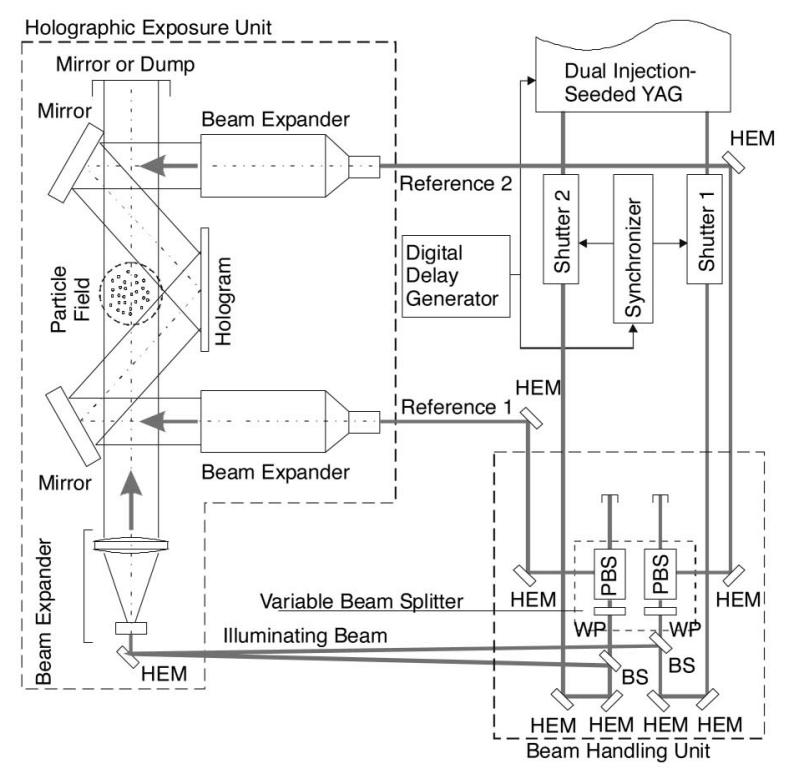

(a)

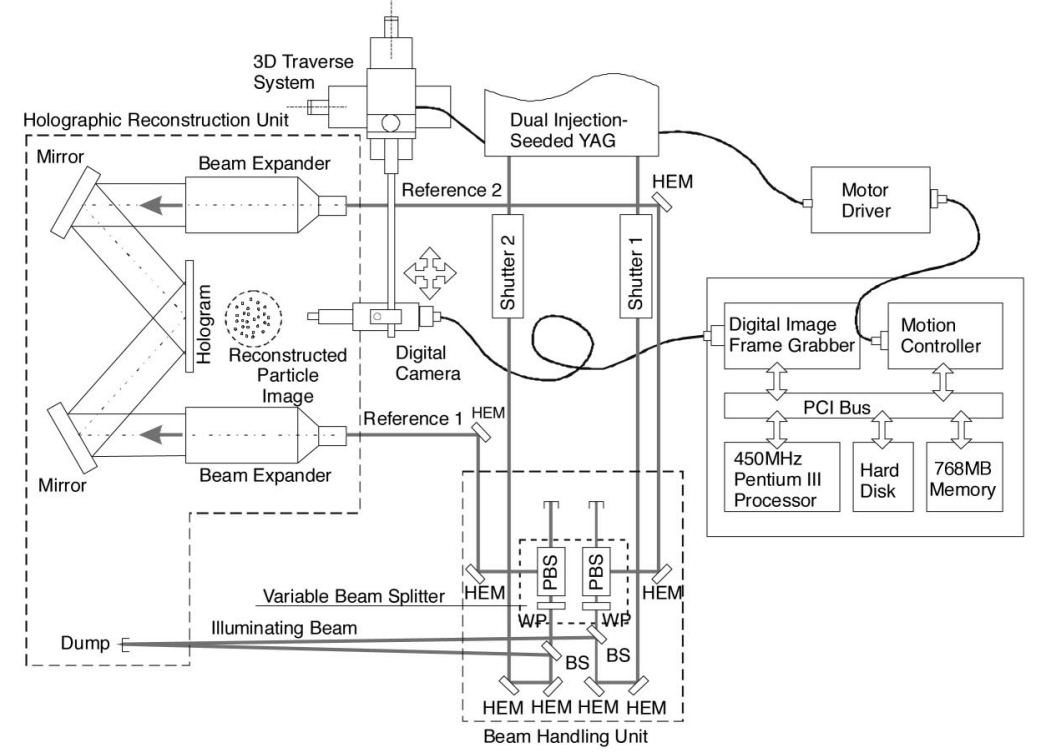

(b)

Fig. 2. Scheme of off-axis holography in the Gemini HPIV system. (a) Recording, (b) reconstruction and data processing. HEM, high-energy mirror; WP, half-wave plate; BS, beam splitter; PBS, polarizing beam splitter; PCI, peripheral component interconnect.

light energy from each beam is reflected and used for illumination. The remaining part of each laser beam is further manipulated by a variable beam splitter and serves as the reference beams at two distinct angles. With this angular-multiplexing scheme, two holograms can be recorded and reconstructed independently.

As shown in Fig. 2(b), the holographic reconstruction system shares the same optics as the recording system to minimize aberrations. The developed hologram is now placed back at the original position with the emulsion side facing opposite to that of the recording, such that each reference beam becomes the complex conjugate of that used in the recording. In this way, an unscrambled real image of the $3 \mathrm{D}$ particle field is reconstructed.

We use the 3D scanning approach to interrogate the reconstructed particle images. A Pentium PC controls the data acquisition. This computer serves as the acquisition node (or the master node in terms of a master-slave programming model) in a distributed computing environment, as well as a stand-alone processing power if no parallel computing facility is available. Image acquisition and camera movement are synchronized with the laser pulses to ensure data integrity. 


\section{B. Extraction of Particle Information}

Given the constraint of information capacity and the desired spatial resolution to measure complex and potentially turbulent flows, it is always preferable to extract as much information (position, size, and displacement) as possible from each individual particle image so that optimal spatial resolution is attained. On the other hand, the extremely complex 3D morphology of the particle images, exacerbated by the speckle noise, poses a great challenge to the retrieval of the particle information. This problem is especially severe when detailed information is needed for categorization of particles.

We have implemented the particle reconstruction by edge detection (PRED) algorithm, a 3D image segmentation and labeling algorithm that extracts the $3 \mathrm{D}$ exterior surface of a particle image based on $2 \mathrm{D}$ segmentation and recursive 3D labeling. ${ }^{30}$ The task of PRED is to travel through the 3D spatial structure of each particle image, pick up as many pixels belonging to the image as possible, and calculate the center coordinate of the image. Essentially, the algorithm consists of two steps: (1) extract all particle 2D boundaries in every image slice (plane) with a hybrid edgeand region-based $2 \mathrm{D}$ segmentation algorithm and (2) collect the $2 \mathrm{D}$ boundaries with a recursive graph traveling algorithm to form $3 \mathrm{D}$ surfaces of the particle images. Interested readers should see Ref. 30 for further details.

Because of the intrinsic aberration, the position where maximum intensity is located (i.e., the focal point) does not represent the image center reliably, especially when faced with speckle noise. Since the intensity concentrates at the vicinity of the particle center, we define the region with intensity exceeding a threshold as the image region, or the image. We use the first moment (center of mass) of intensity enclosed in the image region, i.e., the centroid, to represent the center of the image:

$$
\mathbf{x}_{c}=\frac{\sum_{i} \sum_{j} \sum_{k} I_{i j k} \mathbf{x}}{\sum_{i} \sum_{j} \sum_{k} I_{i j k}}, \quad(i, j, k) \in V_{T},
$$

where $\mathbf{x}_{c}=\left[\begin{array}{lll}x_{c} & y_{c} & z_{c}\end{array}\right]^{T}$ is the coordinate of the centroid, $V_{T}$ is the volume of the image region, $i, j, k$ are the discrete coordinates of the pixels, $\mathbf{x}=\left[\begin{array}{lll}i & j & k\end{array}\right]^{T}$ is the coordinate of any pixel enclosed in $V_{T}$, and $I_{i j k}$ is the intensity value at that pixel.

The extraction accuracy of PRED is benchmarked with a numerically generated planar distribution of $3 \mathrm{D}$ particle images sampled at a $50 \mu \mathrm{m}$ distance. The image intensities are calculated based on Miescattering theory and appropriate pixel sizes. After PRED extracts the particle image centroids, we fit the centroid coordinates to a plane, as shown in Fig. 3(a). The distance between the centroid to the plane represents the extraction error of PRED, whose histogram is plotted in Fig. 3(b). The standard deviation of this error, i.e., the uncertainty in the extraction, is $\sim 5 \mu \mathrm{m}$, in which the axial component dominates. Here we specify the overall uncertainty

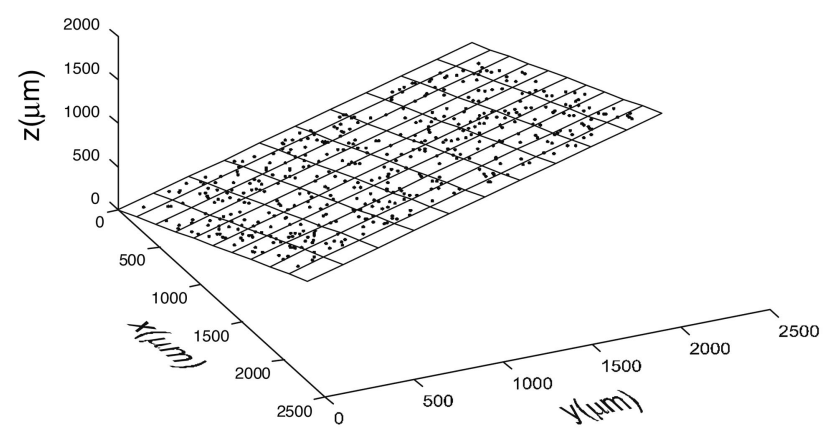

(a)

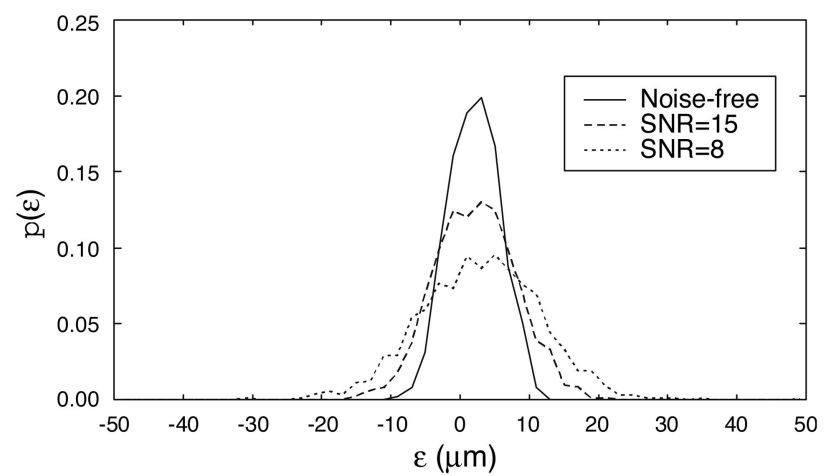

(b)

Fig. 3. Calibration of PRED algorithm through planar distributed particle images generated by simulations of Mie scattering. (a) Spatial distribution of particle centroid extracted by PRED. (b) Statistics of the coordinate errors at various SNRs. Results are averages calculated from ten tests: A total of 500 particle images is generated in each test, and on average 480 particles are extracted by PRED.

(worst case) instead of three separated components. This also applies to the calibration in Section 5.

\section{Extraction of Particle Displacements}

We have implemented a concise cross correlation (CCC) algorithm that correlates and tracks 3D particle movement with discrete particle centroid coordinates. ${ }^{14}$ The name came from the fact that particle centroid coordinates are much more concise information than the particle image itself.

The first step of CCC is to calculate the mean displacement (translation) between two groups of particle coordinates through correlation. We assign each particle an imaginary sphere with virtual radius $r$ centered at its centroid location, and define a correlation kernel function between the $i$ th particle in the first group and $j$ th particle in the second group as

$$
\begin{aligned}
& \Gamma_{i j}(x, y, z ; r) \\
& \quad=\exp \left[-\frac{\left(x-x_{i}+x_{j}\right)^{2}+\left(y-y_{i}+y_{j}\right)^{2}+\left(z-z_{i}+z_{j}\right)^{2}}{2 r^{2}}\right] .
\end{aligned}
$$

The correlation function is indeed a summation of all 


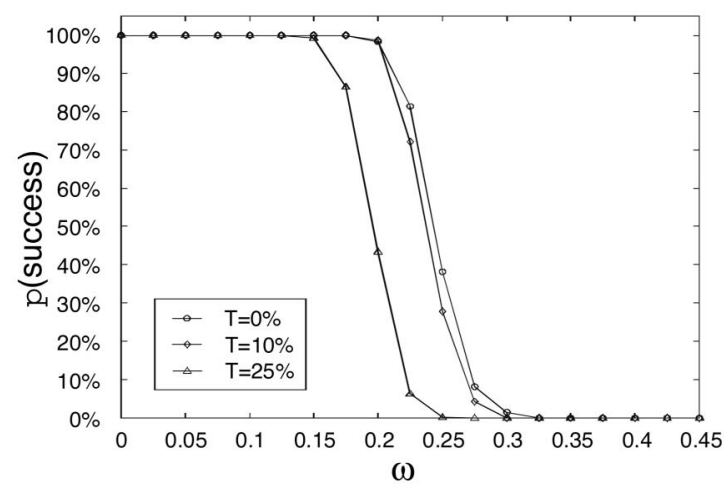

(a)

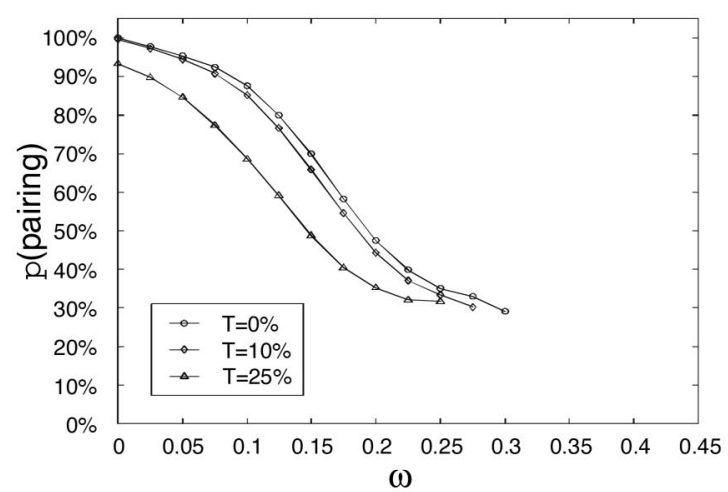

(c)

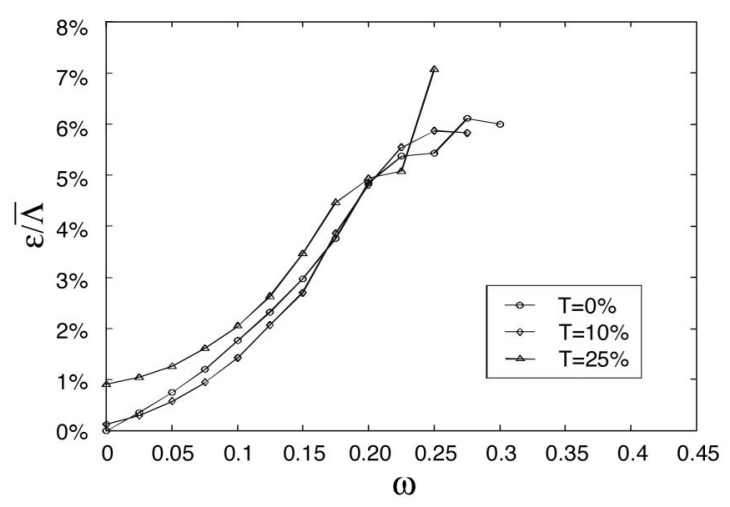

(b)

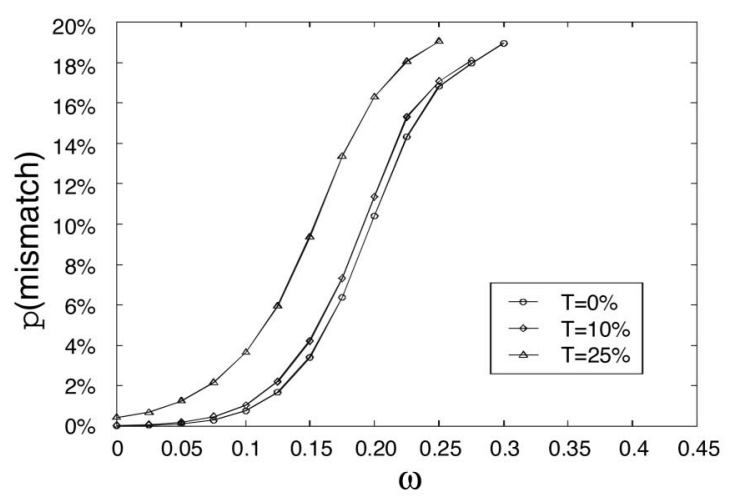

(d)

Fig. 4. Benchmark tests of CCC algorithm based on simulated spiral fluid motion consisting of a linear translation $T$ plus a solid-body rotation $\omega_{1}$ in a volume of $D \times D \times D$. (a) Probability of successful correlations as a function of rotation angle $\omega$ for different levels of translation T. (b) Relative error in correlation results. (c) Probability of paired particles among all particles in one interrogation cell. (d) Percentage of erroneously matched centroids among all paired centroids $\omega$ is expressed in radians. $T$ is expressed in percentage of $D$.

the individual correlation kernel functions:

$$
C(x, y, z)=\sum_{i=1}^{N} \sum_{j=1}^{N} \Gamma_{i j}(x, y, z ; r)
$$

whose highest peak in 3D space represents the mean displacement between the two groups of particles. The virtual radius $r$ serves as the size of particle images in a fast-Fourier-transform-based correlation. ${ }^{28}$ This artificially assigned, adjustable particle size is the key to the great robustness of CCC against gradients of displacement or velocity. The value of $r$ is determined empirically, and the correlation results are robust as long as $r$ is larger than the average amplitude of the displacement gradient within the volume under investigation. The correlation is more robust against displacement gradients with larger $r$ values. On the other hand, a larger $r$ value would slightly increase the correlation error by a few percent. This 3D correlation is properly decomposed into three one-dimensional correlations performed in the space domain for a significant speed-up. After the correlation, CCC next individually pairs each particle based on the information obtained from the first step and a shortest distance classification criterion. The correlation error is largely eliminated by the pairing step, although such an error results in an increased probability of incorrect pairing.

We benchmark the performance of CCC with spiral motions (translation $T$ plus solid-body rotation $\omega$ ) of numerically dispersed particles in a volume-sized $D \times D \times D$. Figure 4 demonstrates the benchmark results showing the robustness of CCC against various combinations of translations and deformations. Through optimization of the virtual radius, CCC handles velocity gradients much better than traditional 3D fast-Fourier-transform based correlation. ${ }^{31}$

\section{Distributed Parallel Processing}

Processing of HPIV data (extraction of information from 3D particle images) represents a major challenge in the development of practical HPIV instruments. The vast amount of image data (often more than 100 Gbytes of raw image data per hologram) takes more than $50 \mathrm{~h}$ for a single $600 \mathrm{MHz}$ Pentiumgrade computer to process just one snapshot. Experiments involving multiple snapshots can produce tens or even hundreds of holograms. This memoryand computation-intensive task calls for a parallel computing architecture to bring the total processing time down to a few hours. 


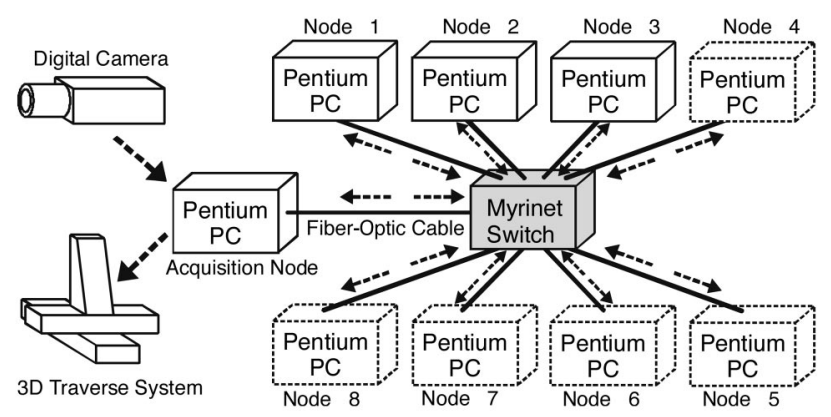

(a)

$\begin{aligned} & \text { Master/ACquisition Process } \\ & \text { Main } \\ & \text { Thread }\end{aligned}$ ACQ
Agent Thread \#1

(b)

Fig. 5. Distributed parallel computing cluster in the Gemini HPIV system. (a) Hardware infrastructure. The current system implemented the acquisition node and three processing nodes. (b) Timing of parallel operations. The design goal was to maximize the overlap among the acquisition, communication, and computation.

On the basis of our initial experimentation with a parallel version of the processing algorithm, ${ }^{32}$ we have recently implemented a parallel computing infrastructure with commodity Pentium PCs connected with a high-speed, low-latency network (Myrinet) to satisfy the computing needs. Because of the on-board communication processor on board the Myrinet interface card, an intensive communication task is offloaded from the main processor in each computing node, making the computing and acquisition operations largely overlapped with the communication.

Figure 5(a) depicts the hardware infrastructure of the distributed parallel processing system. The acquisition node controls the $3 \mathrm{D}$ traverse system to scan the 3D image volume and acquires image data from the digital camera. After image data for one interrogation cell (IC) is collected, the acquisition computer dispatches the data to one of the processing nodes through the Myrinet switch. The 1.28 Gbyte/s data rate of Myrinet ensures prompt and efficient distribution of image data among the processing nodes. The processing nodes send the results back to the acquisition node after they finish their tasks. Figure 5(b) shows the timing of the parallel computing.

The software is based on a master-slave model, with the program running on the acquisition node serving as the master and the programs running on

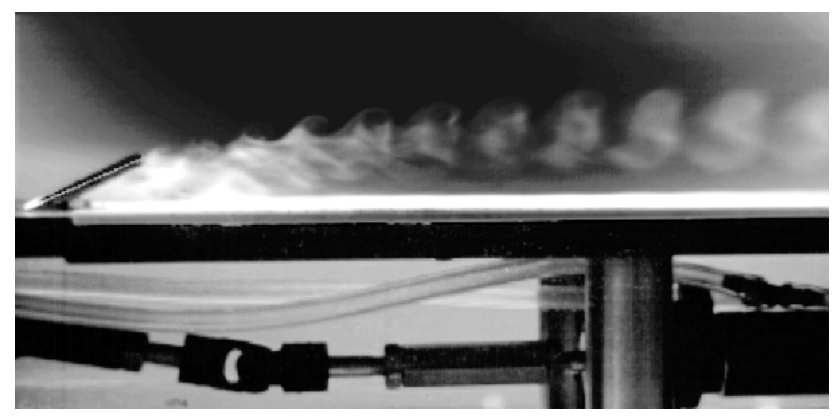

Fig. 6. Flow visualization of vortex shedding based on phaselocked video imaging. Shown is the intensity average of 100 images phase locked with the excitation of the flow. These structures would smear out after the averaging if the flow is not phase locked.

the processing nodes serving as slaves. We use the standard message-passing interface (MPI) for interprocess communication support. The master process continuously scans (through the traverse system) the $3 \mathrm{D}$ image field one IC after another and dispatches the acquired image slices to the slave process, which is a stand alone MPI program implementing the PRED and CCC algorithm.

\section{Phase-Locked Holographic Particle Image Velocimetry Measurement}

The ultimate goal of HPIV is to provide both temporally and spatially resolved $3 \mathrm{D}$ velocity measurements. With the Gemini HPIV system and the implementation of the enhanced processing scheme, for the first time to our knowledge this goal has become tangible. However, the lack of a proper recording media transport system at the present stage prevents us from carrying out such a type of measurements. To demonstrate the capability of the Gemini HPIV system, we instead perform a phase-locked measurement of a flow passing a wall-mounted tab, where the measurement is synchronized with the flow phenomena, and the dynamics of the coherent structure are revealed at a sequence of phases.

To phase lock the coherence structure of the flow with the laser pulses, we first build an active tab, which is driven through a linear solenoid. With a proper driving signal to the solenoid, the tab introduces a small amount of disturbances to the flow at the tab tip. The disturbances are precisely synchronized with the laser pulses at a frequency very close to the natural frequency of the vortex shedding, so that the coherent structure of the flow will be attracted to the excitation frequency and synchronized to the laser pulses. With an accurate delay between the disturbances and the laser pulses, we can record the flow structure at different phases. Because the natural frequency of the tab wake is much lower than the frequency of the laser pulses $(10 \mathrm{~Hz})$, we use a dividing delay generator to produce the driving signal so that the disturbances introduced to the flow are a subharmonic of the laser frequency. Laser Doppler velocimetry (LDV) measurement shows that the flow has a natural frequency of $1.3 \mathrm{~Hz}$. 


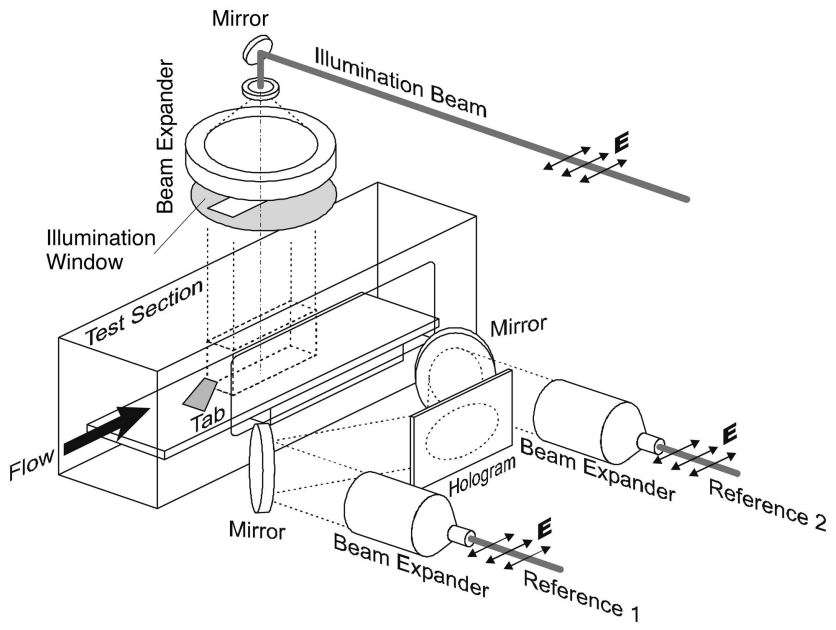

Fig. 7. Optical setup for the HPIV measurement of flow passing a wall-mounted tab. Note that a horizontal polarization configuration is used for optical access.

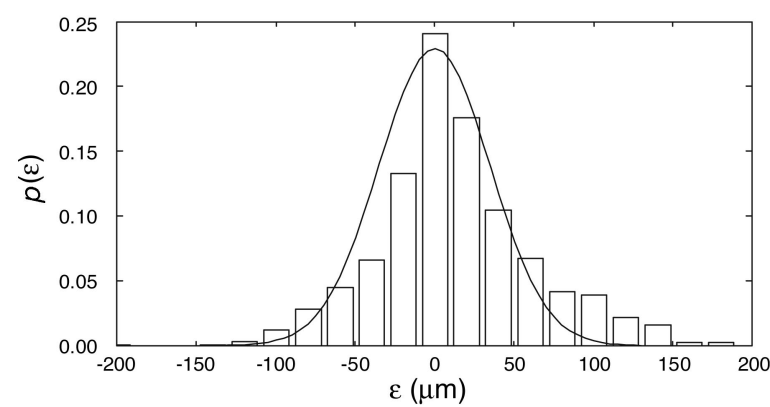

Fig. 8. Calibration of particle centroid uncertainty. A total of 2251 particles was extracted. The solid curve is a best-fit normal probability distribution for reference. The overall uncertainty is $46 \mu \mathrm{m}$.

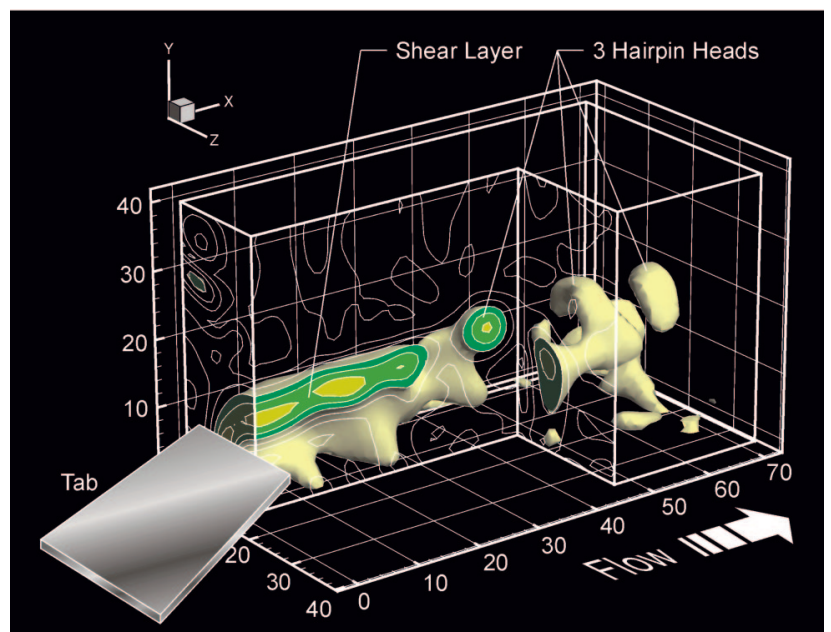

Fig. 9. (Color online) Shown is the 3D vorticity isosurface of the HPIV measured flow at one instant. We cut out a portion of the data volume to show the inner vorticity contour. Threshold for the isosurface is $0.5 \omega_{m}$.

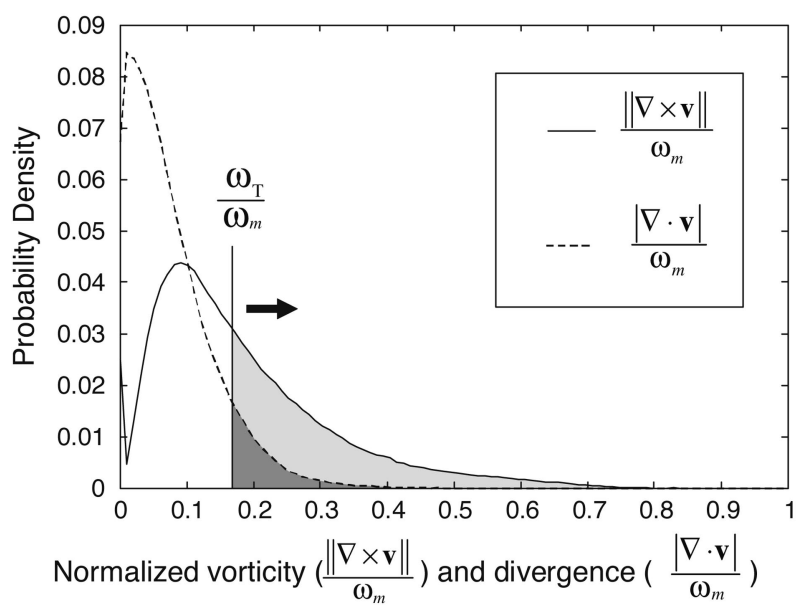

(a)

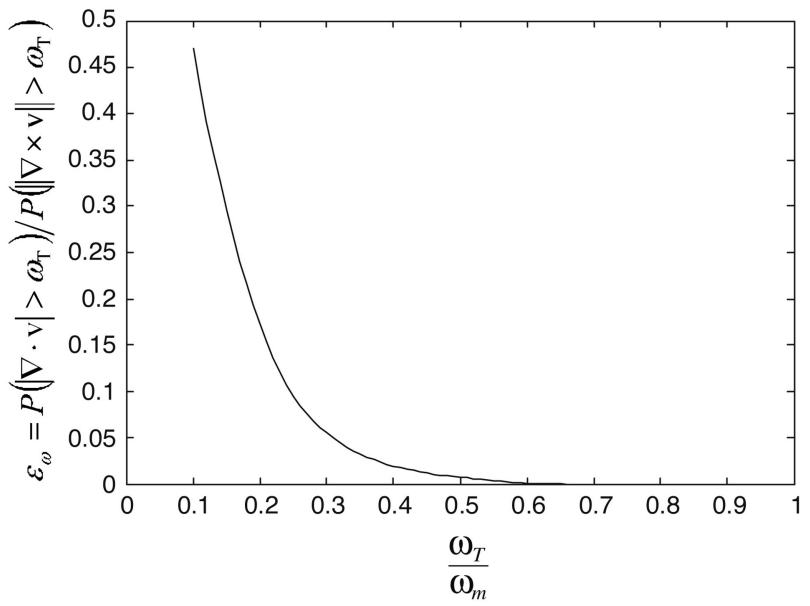

(b)

Fig. 10. Statistics of residual flow divergence (an indicator of errors in experimental data) compared with statistics of flow vorticity. (a) Probability density functions of divergence and vorticity magnitude normalized by maximum vorticity value $\omega_{m}$. The darker shaded area is $P\left(|\nabla \cdot \mathbf{v}|>\omega_{T}\right)$, and the lighter shaded area is $P\left(\|\nabla \times \mathbf{v}\|>\omega_{T}\right)$. (b) The relative vorticity error $\varepsilon \omega\left(\omega_{T}\right)$ as a function of $\omega_{T}$.

Thus the excitation is introduced at $1.25 \mathrm{~Hz}$, the closest subharmonic of the laser frequency, to phase lock the flow.

Flow visualizations indicate that the flow is properly phase locked with the controlled excitation. Figure 6 shows the average intensity of 100 frames of flow visualization images. Because the flow is synchronized with the illumination pulse, the coherent structures of the flow are clearly identifiable. Without the phase-locked excitation, the natural flow would show no identifiable structure since structures in each snapshot are at different phase and hence cancel out.

Shown in Fig. 7 is the optical setup for the flow measurement. Because of the large size and the horizontal orientation of the test section, which is part of 


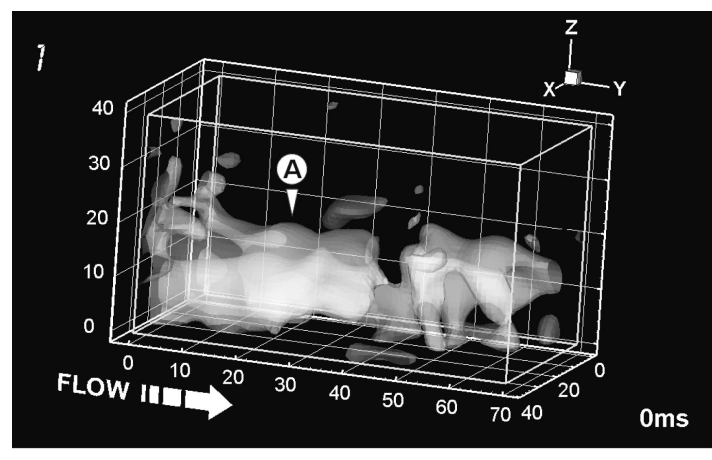

(a)

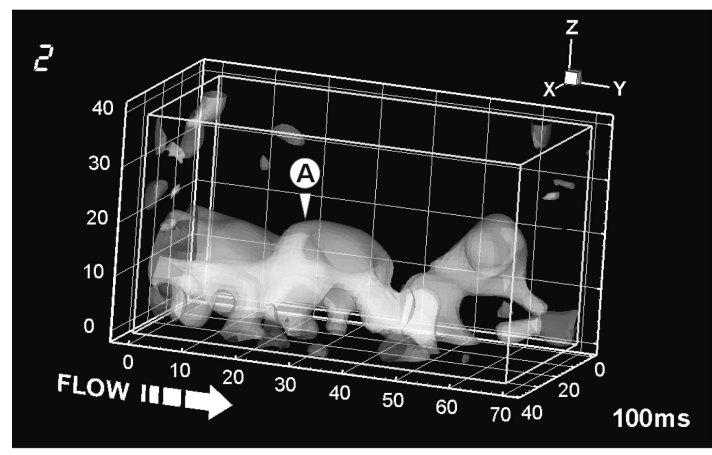

(b)

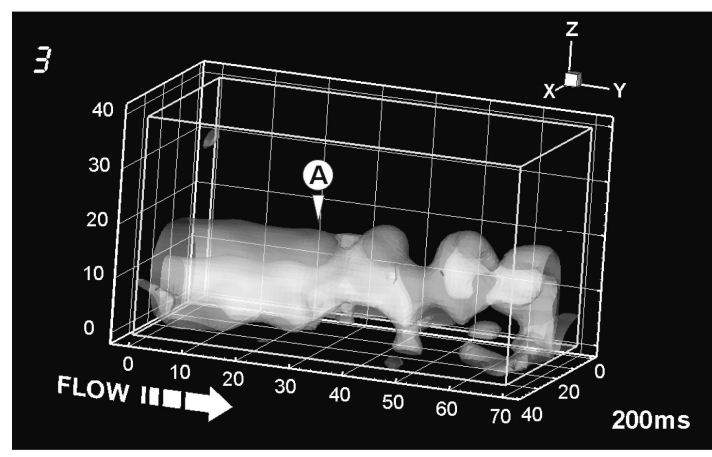

(c)

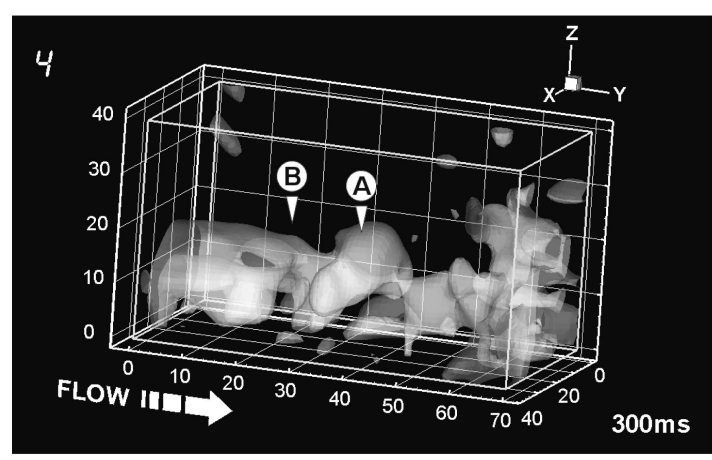

(d)

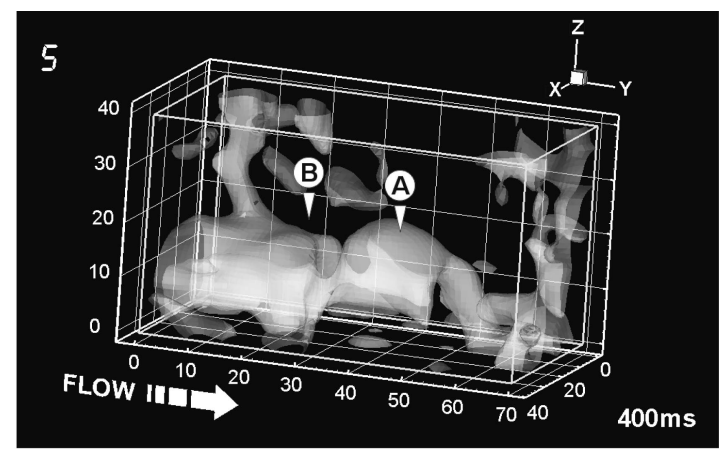

(e)

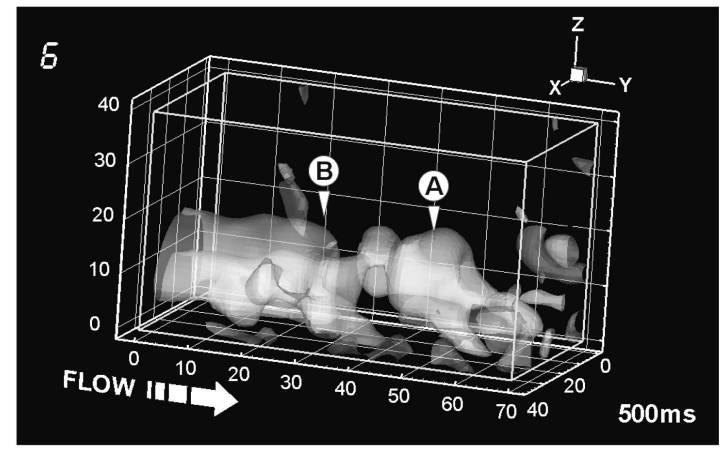

(f)

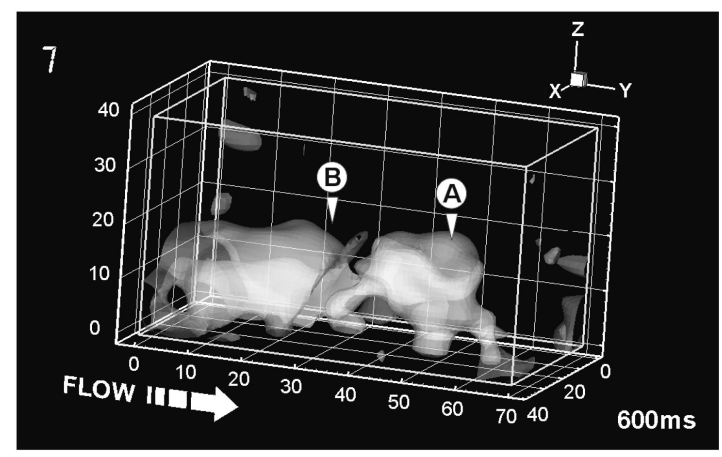

(g)

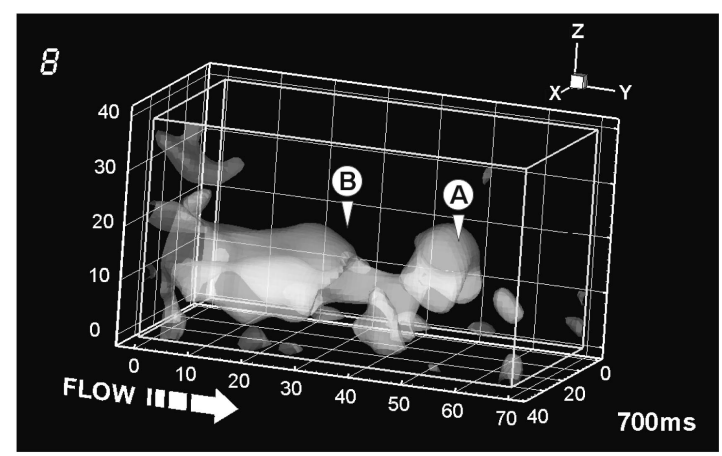

(h)

Fig. 11. Vortex shedding cycle recorded and reconstructed by holographic PIV. The vortex structures are represented by the vorticity isosurface at $\omega_{T}=0.5 \omega_{m}$.

a 6 in. $(15 \mathrm{~cm})$ recirculating water tunnel, we have to introduce the illuminating beam from the top. Therefore, unlike our previous HPIV measurement ${ }^{14}$ where the test section was vertical, here we are dealing with the less preferred horizontal polarization configuration. To counter the decreased fringe visibility in this 
type of configuration, we use fixation-free bleaching ${ }^{33}$ (ferric ethylenediamine tetra-acetic acid III) in the chemical processing of the hologram for better diffraction efficiency and minimal emulsion shrinkage (which is one of the major causes for aberrations in practical holography).

The centroid uncertainty is highly specific to the optical and mechanical configuration. We calibrate this experimental setup by recording a hologram for standard $10 \mu \mathrm{m}$ particles distributed on a flat glass surface and extracting the particle coordinates from the reconstructed images. The deviation of the measured centroids from the regressed plane provides an indication of the measurement uncertainty in the centroid positions. Figure 8 shows the probability distribution of the uncertainty obtained in this manner. The standard deviation of this distribution is $\sim 46 \mu \mathrm{m}$, which is dominated by the axial component. A major part of the uncertainty is due to the aberrations in the hologram, as well as mechanical misalignments and vibrations during 3D scanning. Note that in this calibration we omit the effect due to the aberrations induced by the fluid media because of the small angular aperture.

We record eight holograms in a batch with $100 \mathrm{~ms}$ consecutive phase delay in between. These eight phases cover one complete cycle of the vortex shedding process. On average approximately 50,000 particles are extracted for each snapshot in this measurement, out of which $\sim 20,000$ particles are individually paired to produce velocity vectors. Note that this result is actually worse than our previous experiment reported in Ref. 14 due to the drastically increased recording distance (400 versus $150 \mathrm{~mm}$ ), reduced angular aperture $\left(8.5^{\circ}\right.$ versus $\left.15^{\circ}\right)$, and use of horizontal polarization. The time interval between the double exposure is $8 \mathrm{~ms}$. LDV measurements suggest that the free-stream flow velocity is $\sim 60 \mathrm{~mm} / \mathrm{s}$. Therefore the mean displacement between particle images reconstructed by the hologram is $\sim 480 \mu \mathrm{m}$. Given the $46 \mu \mathrm{m}$ centroid uncertainty from our calibration and the discussion on the velocity uncertainty in Section 4, there is approximately 9.5\% relative velocity error in the paired velocity vectors. We Gaussian interpolate the velocity onto regular grids with a core size of 0.6 IC spacing before further calculations for derivatives. This Gaussian interpolation also serves as a low-pass filter to minimize the effect of the velocity errors, in which on average five pairs participate in the calculation of each vector on a regular grid, reducing the relative error to $4.2 \%$. From this interpolated data field, we calculate its vorticity. Figure 9 shows the vorticity isosurface at a threshold $\omega_{T}=0.5 \omega_{m}$ for one of the eight snapshots. Here $\omega_{m}$ is the maximum magnitude of vorticity in the measurement field. Better means for calculating spatial derivatives from scattered $3 \mathrm{D}$ data exist ${ }^{34}$; however, the simple interpolation suffices for demonstration purposes. The vorticity structures shown in Fig. 9 are consistent with findings from previous flow visualization, PIV, and direct numerical simulations on the tab wake. ${ }^{35-37}$
The choice of vorticity isosurface threshold $\left(\omega_{T}\right)$ must ensure that the errors in the vorticity structures be sufficiently small while major vortex structures are properly revealed. Although it is difficult to give a direct measure on the vorticity error, a comparison between the statistics of residual divergence and vorticity data provides a good estimate. This is because, statistically, random errors contribute to the divergence and vorticity equally, while real velocity gradients do not contribute to the divergence in an incompressible flow. Hence the probability $P(\| \nabla$ $\left.\times \mathbf{v} \|>\omega_{T}\right)$ indirectly measures the amount of error in the vorticity isosurface obtained at $\omega_{T}$. The relative vorticity error, specific to the threshold $\omega_{T}$, can be defined as $\varepsilon_{\omega}\left(\omega_{T}\right)=P\left(|\nabla \cdot \mathbf{v}|>\omega_{T}\right) / P\left(\|\nabla \times \mathbf{v}\|>\omega_{T}\right)$. To illustrate the effect of $\omega_{T}$ on these statistics, in Fig. 10(a) we plot the probability density functions of the magnitude of vorticity and divergence, both normalized by the maximum vorticity $\omega_{m}$. These statistics are performed on data from all eight snapshots. The darker shaded area is $P\left(|\nabla \cdot \mathbf{v}|>\omega_{T}\right.$ ) (a measure of vorticity error), and the lighter shaded area is $P\left(\|\nabla \times \mathbf{v}\|>\omega_{T}\right)$. Clearly, the higher the vorticity isosurface threshold $\omega_{T}$, the less significant the vorticity error is contained within the isosurface. We further plot the relative vorticity error $\varepsilon_{\omega}\left(\omega_{T}\right)$ as a function of $\omega_{T}$ in Fig. 10(b). At $\omega_{T}=0.5 \omega_{m}$, with which Fig. 9 is generated, $\varepsilon_{\omega} \approx 2 \%$.

The whole dynamic cycle of vortex shedding by the tab recorded by holographic PIV is demonstrated in Figs. 11(a)-11(h), where the vortex structures are represented by the vorticity isosurface at $\omega_{T}$ $=0.5 \omega_{m}$. We mark one particular coherent structure $\mathrm{A}$ and follow its spatial-temporal evolution. It can be seen that, structure A originates from the unstable shear layer enveloping the tab edge and evolves into a distinct 3D structure at the instant in Fig. 11(d), while another structure B follows suit. Both A and B are identified with the aid of a video animation to reveal the correspondence of structures across the picture frames. The full dynamic process can best be demonstrated with animated video clips (not included in this paper).

\section{Conclusion}

The accuracy and capacity of HPIV are limited by fundamental imaging issues related to particle scattering and holography, including intrinsic aberration and intrinsic speckle noise. Although often ignored in previous research, the distributions of intensity and phase in the wavefront associated with Mie scattering contribute to system errors, thus resulting in uncertainties in the particle image positions. Such system errors set the primary limit for the accuracy for HPIV measurements. The intrinsic speckle noise, resulting from the self-interference of the 3D dispersed particle images, sets a fundamental limit for the information capacity, which determines the spatial resolution, the measurable spatial extent, and the applicable range of flow Reynolds number in HPIV measurements. These new insights are derived 
from our latest understanding of the imaging characteristics of particle holography.

The latest HPIV system in this report not only incorporates such new insights, but also integrates advanced data processing algorithms and distributed parallel computing technology. For the first time to our knowledge, the goal of both temporally and spatially resolved flow measurements becomes tangible. Although the system does not yet include film transport and is thus not cinematic, we have demonstrated its temporal measurement capability by a series of phase-locked dynamic measurements of instantaneous $3 \mathrm{D}$, three-component velocity fields in a highly $3 \mathrm{D}$ vortical flow.

The authors thank the National Aeronautics and Space Administration for their support through grant NAG3-2464 and the National Science Foundation for their support through grant CTS-9625307. Valuable contributions from Lujie Cao, Andrew G. Bright, and Scott Woodward to the experiments are greatly appreciated.

\section{References}

1. R. J. Adrian, "Particle-imaging techniques for experimental fluid mechanics," Annu. Rev. Fluid Mech. 23, 261-304 (1991).

2. C. E. Willert and M. Gharib, "Digital particle image velocimetry," Exp. Fluids 10, 181-193 (1991).

3. M. P. Arroyo and C. A. Greated, "Stereoscopic particle velocimetry," Meas. Sci. Technol. 2, 1181-1186 (1991).

4. K. Prasad and R. J. Adrian, "Stereoscopic particle image velocimetry applied to liquid flows," Exp. Fluids 15, 49-60 (1993).

5. Y. G. Guezennec, Y. Zhao, and T. J. Gieseke, "High-speed 3-D scanning particle image velocimetry (3-D SPIV)," in Selected Papers from the Seventh International Symposium on Applications of Laser Techniques to Fluid Mechanics, R. J. Adrian, D. F. G. Durão, F. Durst, M. V. Heiter, M. Maeda, and J. H. Whitelaw, eds. (Springer-Verlag, 1996).

6. Ch. Bruecker, "3D scanning PIV applied to an air flow in a motored engine using digital high-speed video," Meas. Sci. Technol. 8, 1480-1492 (1997).

7. J. D. Trolinger, R. A. Belz, and W. M. Farmer, "Holographic techniques for the study of dynamic particle fields," Appl. Opt. 8, 957-961 (1969).

8. B. J. Thompson, "Holographic particle sizing techniques," J. Phys. E 7, 781-788 (1974).

9. P. R. Hobson, "Precision coordinate measurements using holographic recording," J. Phys. E 21, 139-145 (1988).

10. H. Meng and F. Hussain, "In-line recording and off-axis viewing technique for holographic particle velocimetry," Appl. Opt. 34, 1827-1840 (1995).

11. J. O. Scherer and L. P. Bernal, "In-line holographic particle image velocimetry for turbulent flows," Appl. Opt. 36, 93099318 (1997).

12. H. Meng, W. L. Anderson, F. Hussain, and D. Liu, "Intrinsic speckle noise in in-line particle holography," J. Opt. Soc. Am. A 10, 2046-2058 (1993).

13. D. H. Barnhart, R. J. Adrian, C. D. Meinhart, and G. C. Papen, "Phase-conjugate holographic system for high-resolution particle image velocimetry," Appl. Opt. 33, 7159-7169 (1994).

14. Y. Pu and H. Meng, "An advanced off-axis holographic particle image velocimetry (HPIV) system," Exp. Fluids 29, 184-197 (2000).
15. J. Zhang, B. Tao, and J. Katz, "Turbulent flow measurement in a square duct with hybrid holographic PIV," Exp. Fluids 23, 373-381 (1997).

16. A. Lozano, J. Kostas, and J. Soria, "Use of holography in particle image velocimetry measurements of a swirling flow," Exp. Fluids 27, 251-261 (1999).

17. R. Konrath, W. Schröder, and W. Limberg, "Holographic particle image velocimetry applied to the flow within the cylinder of a four-valve internal combustion engine," Exp. Fluids 33, 781-793 (2002).

18. S. F. Herrmann and K. D. Hinsch, "Light-in-flight holographic particle image velocimetry for wind-tunnel applications," Meas. Sci. Technol. 15, 613-621 (2004).

19. K. D. Hinsch and S. F. Hermann, "Holographic particle image velocimetry,” Meas. Sci. Technol. 13, R61-R72 (2002).

20. H. Meng, G. Pan, Y. Pu, and S. H. Woodward, "Holographic particle image velocimetry: from film to digital recording," Meas. Sci. Technol. 15, 673-685 (2004).

21. Y. Pu and H. Meng, "Intrinsic aberrations due to Mie scattering in particle holography," J. Opt. Soc. Am. A 20, 1920-1932 (2003).

22. Y. Pu and H. Meng, "Intrinsic speckle noise in off-axis particle holography," J. Opt. Soc. Am. A 21, 1221-1230 (2004).

23. H. C. van de Hulst, Light Scattering by Small Particles (Dover, 1981)

24. J. M. Coupland and N. A. Halliwell, "Holographic displacement measurements in fluid and solid mechanics: immunity to aberrations by optical correlation processing," Proc. R. Soc. London 453, 1053-1066 (1997).

25. J. W. Goodman, "Film grain noise in wavefront reconstruction imaging," J. Opt. Soc. Am. 57, 493-502 (1967).

26. J. W. Goodman, "Statistical properties of laser speckle patterns," in Laser Speckle and Related Phenomena, J. C. Dainty, ed. (Springer-Verlag, 1975), pp. 9-75.

27. R. L. Panton, Incompressible Flow (Wiley, 1996), p. 770.

28. J. Westerweel, "Fundamentals of digital particle image velocimetry," Meas. Sci. Technol. 8, 1379-1392 (1997).

29. K. Sholes and P. V. Farrell, "Optical alignment-induced errors in holographic particle image velocimetry," Appl. Opt. 39, 5685-5693 (2000).

30. Y. Pu, X. Song, and H. Meng, "Off-axis holographic particle image velocimetry for diagnosing particulate flows," Exp. Fluids 29, S117-S128 (2000).

31. K. Huang, J. Slepicka, and S. S. Cha, "Cross-correlation of threedimensional images for three-dimensional three-component fluid velocity measurements," in Optical Diagnostics in Fluid and Thermal Flow, S. S. Cha and J. D. Trolinger, eds., Proc. SPIE 2005, 655-666 (1993).

32. Y. Pu and D. Andresen, "Distributed processing for cinematic holographic particle image velocimetry," in Proceedings of Eight IEEE International Symposium on High Performance Distributed Computing. (IEEE Press, 1999), pp. 343-344.

33. H. I. Bjelkhagen, Silver-Halide Recording Materials for Holography and Their Processing (Springer-Verlag, 1995).

34. H. Stüer and S. Blaser, "Assessment of spatial derivatives determined from scattered 3D PTV data," Exp. Fluids 30, 492499 (2001).

35. R. Elavarasan and H. Meng, "Flow visualization study of role of coherent structures in a tab wake," Fluid Dyn. Res. 27, 183-197 (2000).

36. W. Yang, H. Meng, and J. Sheng, "Dynamics of hairpin vortices generated by a mixing tab in a channel flow," Exp. Fluids 30, 705-722 (2001)

37. S. C. Dong and H. Meng, "Direct numerical simulation of the mixing tab flow," J. Fluid Mech. 510, 219-242 (2004). 